\title{
Stillbirth risk across pregnancy by size for gestational age in Western Cape Province, South Africa: Application of the fetuses-at-risk approach using perinatal audit data
}

\author{
T Lavin, ${ }^{1}$ PhD, MPH, MIntHlth, BSc Hons; R C Pattinson, ${ }^{2}$ BSc, MB ChB, MMed (O\&G), FGOG (SA), MD, FRCOG; L Nedkoff, ${ }^{3}$ PhD, \\ MPH, GradDipBus, BAppSc; S Gebhardt, ${ }^{4}$ MB ChB, FCOG (SA), MMed (O\&G), Hons BSc (Epidemiol), MD; D B Preen, ${ }^{1}$ BSc Hons, PhD \\ ${ }^{1}$ Centre for Health Services Research, School of Population and Global Health, Faculty of Health and Medical Sciences, University of Western \\ Australia, Australia \\ ${ }^{2}$ South African Medical Research Council Maternal and Infant Health Care Strategies Unit, Department of Obstetrics and Gynaecology, \\ School of Medicine, Faculty of Health Sciences, University of Pretoria, South Africa \\ ${ }^{3}$ Cardiovascular Research Group, School of Population and Global Health, Faculty of Health and Medical Sciences, University of Western \\ Australia, Australia \\ ${ }^{4}$ Department of Obstetrics and Gynaecology, Faculty of Medicine and Heath Sciences, Stellenbosch University and Tygerberg Hospital, \\ Cape Town, South Africa
}

Corresponding author: T Lavin (tina.lavin@uwa.edu.au)

\begin{abstract}
Background. There is little published work on the risk of stillbirth across pregnancy for small-for-gestational-age (SGA) and large-forgestational (LGA) pregnancies in low-resource settings.

Objectives. To compare stillbirth risk across pregnancy between SGA and appropriate-for-gestational-age (AGA) pregnancies in Western Cape Province, South Africa (SA).

Methods. A retrospective audit of perinatal mortality data using data from the SA Perinatal Problem Identification Program was conducted. All audited stillbirths with information on size for gestational age $(N=677)$ in the Western Cape between October 2013 and August 2015 were included in the study. The Western Cape has antenatal care (ANC) appointments at booking and at 20, 26, 32, 34, 36, 38 and 41 (if required) weeks' gestation. A fetuses-at-risk approach was adopted to examine stillbirth risk ( $28-42$ weeks' gestation, $\geq 1000 \mathrm{~g})$ across gestation by size for gestational age (SGA $<10$ th centile Theron growth curves, LGA $>90$ th centile). Stillbirth risk was compared between SGA/LGA and AGA pregnancies.

Results. SGA pregnancies were at an increased risk of stillbirth compared with AGA pregnancies between 30 and 40 weeks' gestation, with the relative risk (RR) ranging from 3.5 (95\% confidence interval (CI) 1.6 - 7.6) at 30 weeks' gestation to $15.3(95 \% \mathrm{CI} 8.8$ - 26.4) at 33 weeks' gestation $(p<0.001)$. The risk for LGA babies increased by at least 3.5 -fold in the later stages of pregnancy (from 37 weeks) $(p<0.001)$. At 38 weeks, the greatest increased risk was seen for LGA pregnancies (RR 6.6, 95\% CI $3.1-14.2 ; p<0.001$ ).

Conclusions. There is an increased risk of stillbirth for SGA pregnancies, specifically between 33 and 40 weeks' gestation, despite fortnightly ANC visits during this time. LGA pregnancies are at an increased risk of stillbirth after 37 weeks' gestation. This high-risk period highlights potential issues with the detection of fetuses at risk of stillbirth even when ANC is frequent.
\end{abstract}

S Afr Med J 2019;109(12):927-933. https://doi.org/10.7196/SAMJ.2019.v109i12.13764

In low- and middle-income countries (LMICs), $\sim 25 \%$ of infants born are small for gestational age (SGA). ${ }^{[1]}$ The fetus may be small but healthy, or the condition may be due to pathological growth failure (intrauterine growth restriction (IUGR)). ${ }^{[2]}$ Compared with appropriate-for-gestational-age (AGA) infants, SGA infants are at increased risk of perinatal mortality, ${ }^{[3]}$ including a 10 -fold increased risk of stillbirth. ${ }^{[4]}$ SGA babies are also at increased risk of neonatal morbidity such as respiratory distress syndrome $\mathrm{e}^{[5]}$ and bronchopulmonary dysplasia. ${ }^{[6]}$

Studies to date have indicated that there may be a critical gestational period for increased mortality for SGA babies. ${ }^{[3,7-9]}$ A South African (SA) study found that $~ 50 \%$ of SGA stillbirths occur between 33 and 37 weeks' gestation ${ }^{[9]}$ while work in the UK demonstrated a critical period for SGA stillbirth between 28 and 36 weeks. ${ }^{[7]}$ Other studies have found an increased risk for SGA pregnancies to be present at all gestational ages, ${ }^{[3]}$ with the risk of stillbirth for SGA babies increasing with advancing gestational age. ${ }^{[8]}$
Most obstetric clinics have a programme for identifying SGA fetuses antenatally because of their increased risk of fetal complications. ${ }^{[4]}$ SGA fetuses that are not detected during the antepartum period are at a four-fold increased risk of serious fetal complications compared with SGA fetuses detected before delivery, making the detection and management of such infants crucial to prevent adverse outcomes. ${ }^{[4]}$ However, many challenges with detection are present in both highrisk and low-resource settings. Barriers to detection of SGA may be the limited effectiveness of fundal height assessment, ${ }^{[10]}$ as well as the timing of routine ultrasound. ${ }^{[1]}$ A recent French study ( $N=14$ 100) highlighted difficulties with the detection of fetal growth restriction even in the presence of obstetric and medical risk factors for the condition, and suggested that better risk assessment could improve antenatal identification. ${ }^{[12]}$

In SA $\sim 50 \%$ of stillbirths occur in a seemingly healthy mother who does not present with any clinical complications antenatally. ${ }^{[13]}$ Maternal complications are also not increased in SGA stillbirths 
compared with AGA and large-for-gestational-age (LGA) stillbirths. ${ }^{[9]}$ Recently a lack of antenatal care (ANC) in the third trimester of pregnancy has been associated with increased stillbirth risk during this time, ${ }^{[14-16]}$ but the risk for SGA babies specifically was not examined. In SA provinces where ANC appointments are not routinely scheduled between 32 and 38 weeks, there is an increased risk of stillbirth specifically during this time; however, in Western Cape Province, where fortnightly visits commonly occur between 32 and 38 weeks, there is no increased risk for overall stillbirth during this time. ${ }^{[16]}$

\section{Objectives}

Given the increased risk of stillbirth specifically for SGA infants observed in other studies, we sought to explore how stillbirth risk differed for SGA, AGA and LGA infants in the Western Cape, where fortnightly ANC visits occur during the third trimester, to determine whether SGA infants were at increased risk of stillbirth.

\section{Methods}

Data from the SA Perinatal Problem Identification Program (PPIP) were used to analyse stillbirths ( $\geq 1000 \mathrm{~g}$ and $\geq 28$ weeks' gestation) and total number of live births occurring between October 2013 and August 2015 in the Western Cape. A detailed description of PPIP can be found elsewhere. ${ }^{[16-17]}$ In brief, data for the Western Cape are audited for $>90 \%$ of perinatal deaths by the PPIP. The programme allowed for comparisons between SGA, AGA and LGA babies as derived from SA (Theron) growth charts. ${ }^{[18,19]}$ SGA was defined as neonates $<10$ th centile for gestational age and LGA as $>90$ th centile. Data were included for 28 - 42 weeks' gestation, where previous studies have reported high stillbirth risk periods. ${ }^{[3,7,8]}$ Gestational age was calculated based on the date of the last menstrual period, ultrasound or clinical examination, and cases were excluded if the gestational age was unknown or if the estimated age was considered uncertain. No hierarchy was employed in determining gestational age. Only singleton pregnancies in women who had reported receiving ANC were included. After exclusion of deaths before 28 weeks (and after 42 weeks), deaths with unknown or uncertain gestation, multiple pregnancies and women who had not received ANC, the number of deaths used for analysis was 677 .

The data used for the analysis were unidentifiable secondary data collected routinely as part of a clinical audit on reporting of perinatal deaths and collected with permission from the SA National Department of Health. This secondary analysis was approved by the PPIP technical task team of the South African Medical Research Council and the University of Western Australia Human Research Ethics Committee (ref. no. RA/4/1/7955).

\section{Statistical analysis}

SPSS version 24 (IBM Corp., USA) and SAS statistical software version 9.4 (SAS Institute, USA) were used for statistical analyses.

\section{Stillbirth rate}

The stillbirth rate was calculated using the number of audited stillbirths/the total number of births identified in the PPIP data, and expressed as stillbirths per 1000 births. The overall incidence of stillbirth for the study period was calculated, as well as the cumulative stillbirth rate at each gestational age (weekly at 28 - 42 weeks).

\section{Stillbirth risk}

A fetuses-at-risk (FAR) approach ${ }^{[20,21]}$ was adopted using Yudkin et al.'s ${ }^{[21]}$ accepted method of stillbirth risk calculation, and was appropriate for this analysis. ${ }^{[21,22]}$ Yudkin et al.'s ${ }^{[21]}$ approach considers the number of fetuses still in utero as the population at risk. These methods have been published previously for similar work with our data. ${ }^{[16]}$ As there were no data available in the Western Cape regarding the number of SGA/AGA/LGA infants born alive at each gestational age, we used data from the Mamelodi subdistrict to estimate the proportion of SGA/AGA/LGA live births occurring at each gestational age in the Western Cape. This involved several steps. (i) Data from the Mamelodi subdistrict were used to ascertain the proportion of live births at each gestation that were SGA/AGA/LGA. As few stillbirths occurred in the 28 - 32-week period $(<10 \%)$, these data were combined into a single category. This distribution was applied to the Western Cape data for known live births. (ii) The number of SGA fetuses still in utero at each gestational age was calculated by subtracting the number of SGA live births and the number of SGA stillbirths at each gestational age from the total number of SGA births (live and stillbirths) for the entire pregnancy period. This was repeated for AGA and LGA pregnancies. (iii) Stillbirth risk was calculated for SGA/AGA/LGA pregnancies separately, e.g. stillbirth risk for SGA pregnancies at 28 weeks $=$ (no. SGA stillbirths at 28 weeks/no. SGA fetuses still in utero at 28 weeks) $\times 1000$, expressed as the number of SGA stillbirths per 1000 SGA fetuses still in utero. (iv) The crude relative risk (RR) between SGA and AGA pregnancies, and LGA and AGA pregnancies, was calculated at each gestational age. $(v)$ Sensitivity analysis was conducted as outlined below.

\section{Hazard ratio}

A proportional hazard approach was adopted to compare stillbirth risk across gestation between SGA and LGA pregnancies with AGA pregnancies. The Cox regression model used an interaction term for size for gestational age multiplied by time across gestation (grouped as a factor) across the gestational period. The time periods adopted for the Cox regression model were $<33$ weeks, 34 - 36 weeks, 37 weeks, 38 weeks, 39 weeks and $40+$ weeks. Hazard ratios (HRs) and $95 \%$ confidence intervals (CIs) were calculated at each time point for comparisons between size-for-gestational-age groups.

\section{Sensitivity analysis}

As the proportion of SGA/AGA/LGA live births occurring at each gestational age was derived from Mamelodi subdistrict, a sensitivity analysis was conducted to ensure that the distribution of SGA/AGA/ LGA occurring across pregnancy was a reasonable and valid approach for the calculation of stillbirth risk. Here we applied the same method as described for the Mamelodi data using the proportion of SGA/ non-SGA live births at each gestation from a US cohort. ${ }^{[3]}$ As the US cohort did not contain information on LGA births, we compared SGA live births with non-SGA births (LGA + AGA).

\section{Results}

There were 1802 stillbirths ( $\geq 1000 \mathrm{~g}$ and $\geq 28$ weeks' gestation) and 119598 live births during the study period.

\section{Clinical characteristics}

The maternal characteristics for SGA/AGA/LGA pregnancies are shown in Table 1. There were no statistically significant differences between the characteristics of SGA/AGA/LGA pregnancies according to maternal age, parity, HIV status or syphilis infection.

\section{Stillbirth rate}

The overall cumulative incidence of stillbirth for the study period was 5.7 per 1000 births. SGA pregnancies had the highest cumulative incidence of stillbirth at 21.4 deaths per 1000 births, followed by 


\begin{tabular}{|c|c|c|c|c|c|}
\hline & SGA $(N=257), n(\%)$ & $p$-value ${ }^{*}$ & AGA $(N=429), n(\%)$ & LGA $(N=55), n(\%)$ & $p$-value ${ }^{*}$ \\
\hline Parity & & 0.078 & & & 0.115 \\
\hline Primipara & $105(40.9)$ & & $158(36.8)$ & $18(32.7)$ & \\
\hline Multipara & $137(53.3)$ & & $256(59.7)$ & $33(60.0)$ & \\
\hline Grand multipara & $6(2.3)$ & & $3(0.7)$ & $2(3.6)$ & \\
\hline Unknown & $9(3.5)$ & & $12(2.8)$ & $2(3.6)$ & \\
\hline HIV status & & 0.071 & & & 0.393 \\
\hline Positive & $59(23)$ & & $73(17)$ & $7(12.7)$ & \\
\hline Negative & $198(77)$ & & $349(81.4)$ & $48(87.3)$ & \\
\hline Unknown & 0 & & $7(1.6)$ & 0 & \\
\hline Syphilis & & 0.324 & & & 0.968 \\
\hline Positive & $13(5.1)$ & & $15(3.5)$ & $2(3.6)$ & \\
\hline Negative & $243(94.6)$ & & $410(95.6)$ & $53(96.4)$ & \\
\hline Unknown & $1(0.4)$ & & $4(0.9)$ & 0 & \\
\hline Maternal age (years) & & 0.099 & & & 0.779 \\
\hline $15-24$ & $95(37)$ & & $159(37.1)$ & $19(34.5)$ & \\
\hline $25-34$ & $113(44)$ & & $215(50.1)$ & $27(49.2)$ & \\
\hline $35-44$ & $46(17.9)$ & & $49(11.4)$ & $8(14.5)$ & \\
\hline$\geq 45$ & 0 & & $1(0.2)$ & 0 & \\
\hline Unknown & $3(1.2)$ & & $5(1.2)$ & $1(1.8)$ & \\
\hline
\end{tabular}

LGA (11.1 per 1000$)$ and AGA pregnancies (3.7 per 1000$)$.

Overall, the stillbirth rate and AGA stillbirth rate increased across pregnancy to peak at 31 weeks (73.2 and 54.9 deaths per 1000 births, respectively), at which time the rate decreased steadily to 40 weeks to 3.2 per 1000 and 2 per 1000 , respectively (Fig. 1). For SGA pregnancies, the stillbirth rate increased to 33 weeks, then decreased steadily to 35 weeks. At 35 weeks ( 2 000 g), the stillbirth rate decreased until term. For LGA pregnancies, the stillbirth rate was high at earlier gestations ( 37 - 90 deaths per 1000 births) then decreased rapidly to 32 weeks, where it continued to decrease to 37 weeks and remained very low $(<1$ per 1000$)$ until term (Fig. 1).

\section{Stillbirth risk}

For AGA pregnancies, the stillbirth risk was low $(<0.6$ per 1000$)$ and stable across pregnancy, then increased at 41 weeks as expected (Fig. 2). SGA babies had a 3.5 - 5.8fold increased risk of stillbirth compared with AGA babies between 30 and 40 weeks' gestation. SGA pregnancies also had a rapid increase in stillbirth risk at 41 weeks. The risk for LGA babies increased in the later stages of pregnancy (from 37 weeks), then increased rapidly at 41 weeks. Table 2 presents the RR and 95\% CI of stillbirth for SGA and LGA pregnancies compared with AGA pregnancies at each gestational age.

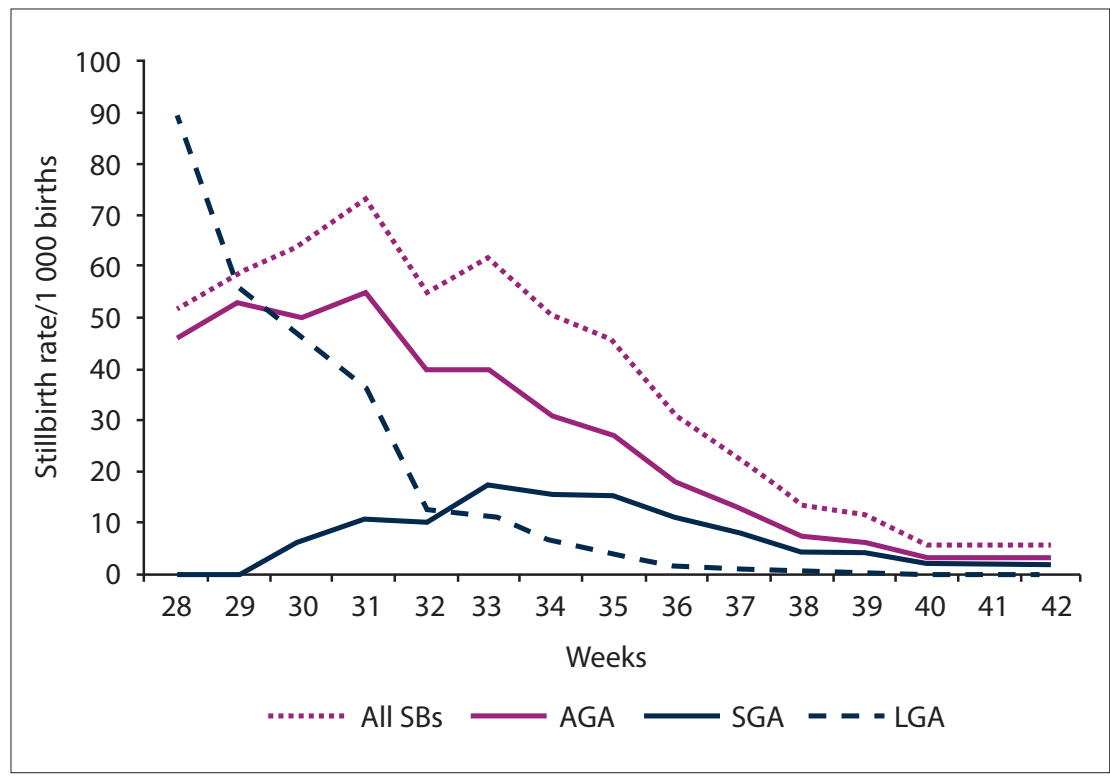

Fig. 1. Cumulative $S B$ rate across pregnancy for all $S B$ s and $A G A, S G A$ and $L G A S B$ in the Western Cape, South Africa, October 2013 - August 2015. (SB = stillbirth; AGA = appropriate for gestational age; $S G A=$ small for gestational age; $L G A=$ large for gestational age.)

For SGA pregnancies, the greatest increased risk was observed at 33 weeks (RR 15.3, 95\% CI 8.8 - 26.4; $p<0.001)$, while for LGA pregnancies the greatest increased risk was observed at 38 weeks (RR 6.6, 95\% CI 3.1 14.2; $p<0.001)$. The proportional hazards model comparing stillbirth risk between SGA and AGA pregnancies showed that SGA pregnancies had an HR $>1$ from 35 to 40 weeks $(p<0.05)$ (Fig. 3). For LGA pregnancies, the HR was $>1$ from 37 to 40 weeks $(p<0.05)$.

\section{Sensitivity analysis}

The distribution of SGA live births across gestation was fairly consistent between the cohorts tested. In the US cohort, ${ }^{[3]}$ the proportion of SGA births at each gestation ranged from $9.1 \%$ to $12.6 \%$. In the Mamelodi population, it ranged from $8.0 \%$ to $10.8 \%$ 


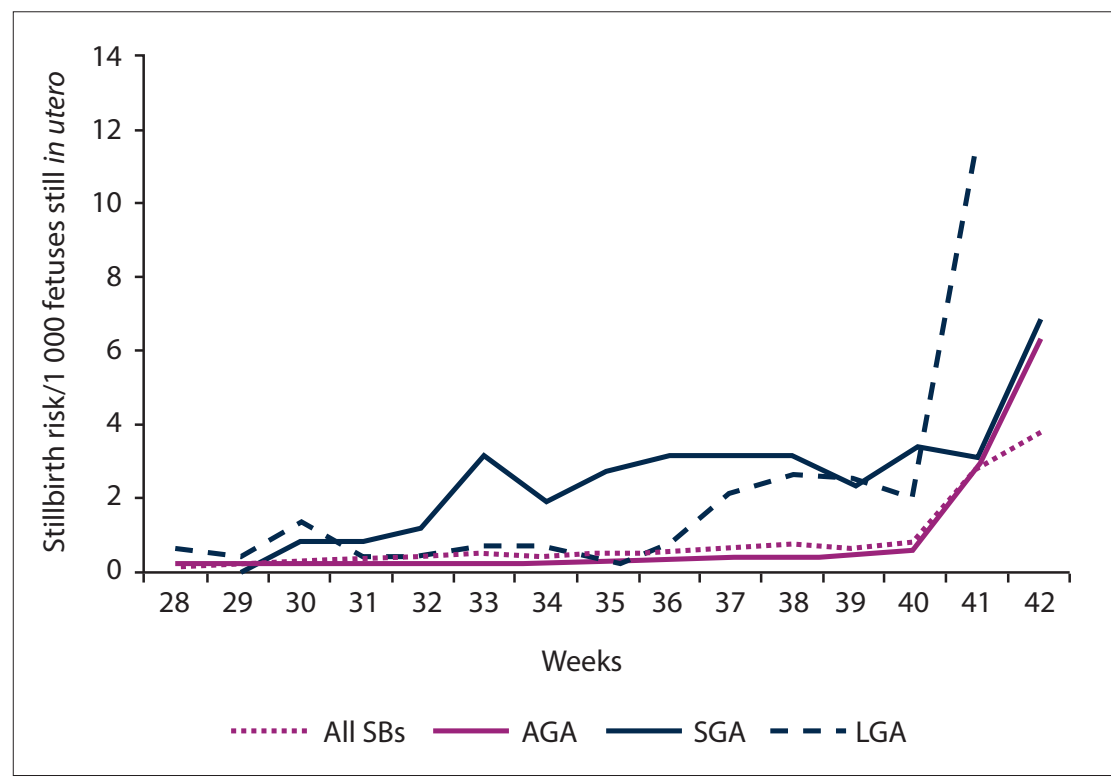

Fig. 2. SB risk across pregnancy for all SBs and AGA, SGA and LGA stillbirths in the Western Cape, South Africa, October 2013 - August 2015. ( $S B=$ stillbirth; AGA = appropriate for gestational age; $S G A=$ small for gestational age; $L G A=$ large for gestational age.)

Table 2. RRs (95\% CI) for stillbirth between SGA and LGA pregnancies compared with AGA pregnancies in the Western Cape, South Africa, October 2013 - August 2015

\begin{tabular}{|c|c|c|}
\hline Gestation (weeks) & $\begin{array}{l}\text { SGA v. AGA*, RR of } \\
\text { stillbirth }(95 \% \mathrm{CI})\end{array}$ & $\begin{array}{l}\text { LGA v. AGA*, RR of } \\
\text { stillbirth }(95 \% \mathrm{CI})\end{array}$ \\
\hline 28 & - & - \\
\hline 29 & - & - \\
\hline 30 & $3.5(1.6-7.6)$ & $5.6(2.3-13.7)$ \\
\hline 31 & $3.5(1.6-7.6)$ & - \\
\hline 32 & $4.1(2.1-7.9)$ & - \\
\hline 33 & $15.3(8.8-26.4)$ & $3.4(1.0-11.3), p=0.049$ \\
\hline 34 & $7.6(4.2-13.6)$ & - \\
\hline 35 & $9.6(5.7-16.1)$ & - \\
\hline 36 & $9.4(5.7-15.3)$ & - \\
\hline 37 & $7.8(4.8-12.6)$ & $5.3(2.4-11.9)$ \\
\hline 38 & $7.8(4.7-12.9)$ & $6.6(3.1-14.2)$ \\
\hline 39 & $5.1(2.8-9.4)$ & $5.6(2.3-13.3)$ \\
\hline 40 & $5.8(3.3-10.0)$ & $3.4(1.2-9.5)$ \\
\hline 41 & - & - \\
\hline 42 & - & - \\
\hline
\end{tabular}

between 34 and 40 weeks, with the highest proportion of SGA live births observed at the later gestational ages $(22.5 \%)$. The overall pattern of stillbirth risk by size for gestational age did not differ significantly between the methods used (Fig. 4). The relative risks of stillbirth between SGA and non-SGA pregnancies only differed by \pm 0.2 for gestations between 28 and 40 weeks with the different methods of estimating the proportion of SGA/non-SGA live births at each gestation (Table 3). For example, the RR for stillbirth at 36 weeks was 8.3 (95\% CI 5.1 -

\section{Discussion}

This study found that SGA pregnancies had a higher cumulative incidence of stillbirth than AGA pregnancies during the study period. SGA pregnancies were at an increased risk of stillbirth throughout pregnancy, particularly between 31 and 40 weeks. LGA pregnancies also had a higher cumulative incidence of stillbirth than AGA pregnancies, with the greatest risk of stillbirth observed during the later stages of pregnancy from 37 weeks.

The FAR approach we used to calculate stillbirth risk revealed important information that would not be uncovered using only the stillbirth rate. In the present study, the cumulative stillbirth rate across gestation showed an increase in the rate to 31 weeks, then a decline from 31 to 41 weeks (Fig. 1). The decline in the stillbirth rate after 31 weeks was expected and consistent with the literature, as it is well known that preterm infants have increased perinatal mortality. ${ }^{[21]}$ However, when the FAR approach was used, a different pattern emerged: early gestational ages carried the lowest risk of stillbirth, with later gestational ages carrying the highest risk. The risk at 41 weeks was $\sim 10$ times that for the preterm period. The reason for this difference lies in the denominator used, as the FAR approach uses the number of fetuses still in utero. By term and postterm dates most babies have been delivered, meaning that the denominator is very small. Although the stillbirth rate is often used as a conventional method to assess the trends and patterns in stillbirth, the present study emphasises the importance of differentiating between stillbirth risk (FAR) and stillbirth rate. In the present study the FAR approach was a more meaningful denominator, as it calculates the chance of death as pregnancy progresses rather than the number of deaths per the number of births that have already occurred.

The finding that stillbirth risk increased in SGA pregnancies with advancing gestational age (Fig. 2) is consistent with numerous other studies that also used the FAR approach..$^{[3,23,24]}$ Rosenstein et al. ${ }^{[24]}$ found that the stillbirth risk for all sizes for gestational age increased steadily after 37 weeks, while another study found that the risk of stillbirth for SGA pregnancies more than doubled after 37 weeks. ${ }^{[23]}$. This same pattern was observed in the present study, where SGA pregnancies had an increased risk of stillbirth from 33 weeks onwards. Pilliod et al. ${ }^{[3]}$ demonstrated a dose-response effect, where SGA babies below the 3rd centile were at greater risk of stillbirth across all gestations compared with AGA 


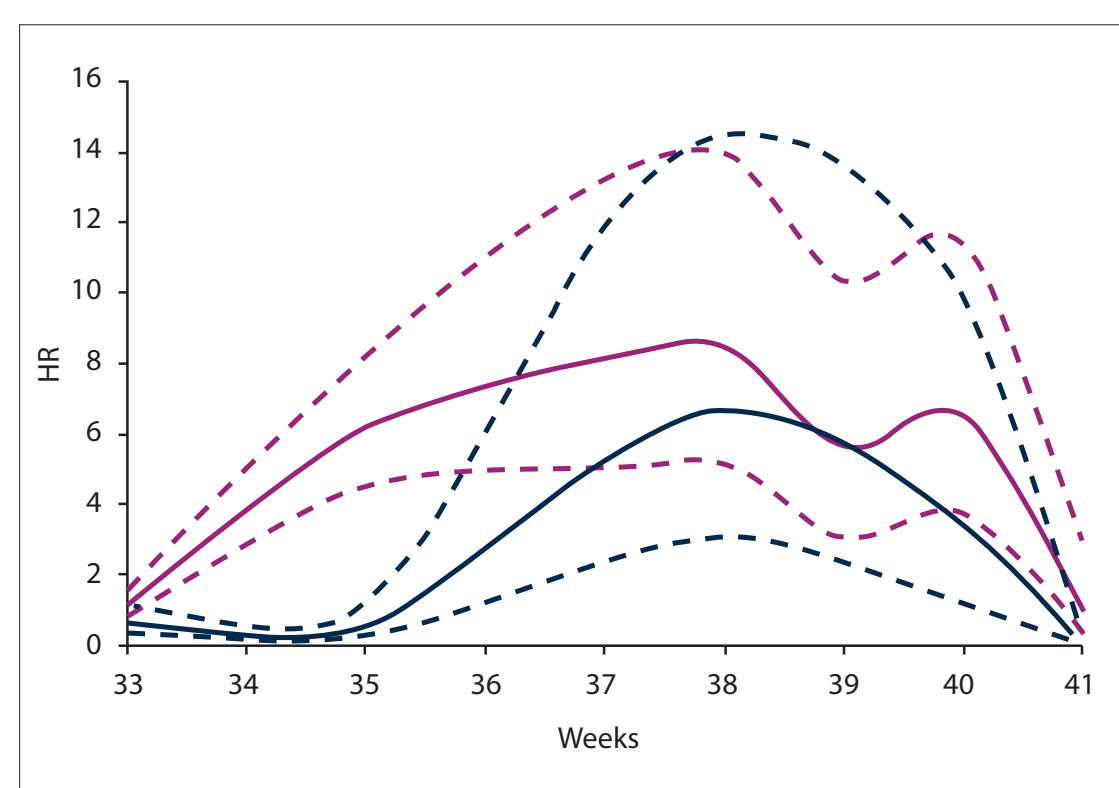

Fig. 3. Estimated HR for stillbirth in the Western Cape, South Africa, October 2013 - August 2015 (purple solid line $=S G A$; black solid line $=L G A$ ) with $95 \%$ confidence intervals (dotted lines) for size for gestational age (relative to $A G A)$ as a function of time $(<33,34-36,37,38,39,40$ and 41 weeks). (HR= hazard ratio; $S G A=$ small for gestational age; $L G A=$ large for gestational age; $A G A=$ appropriate for gestational age.)

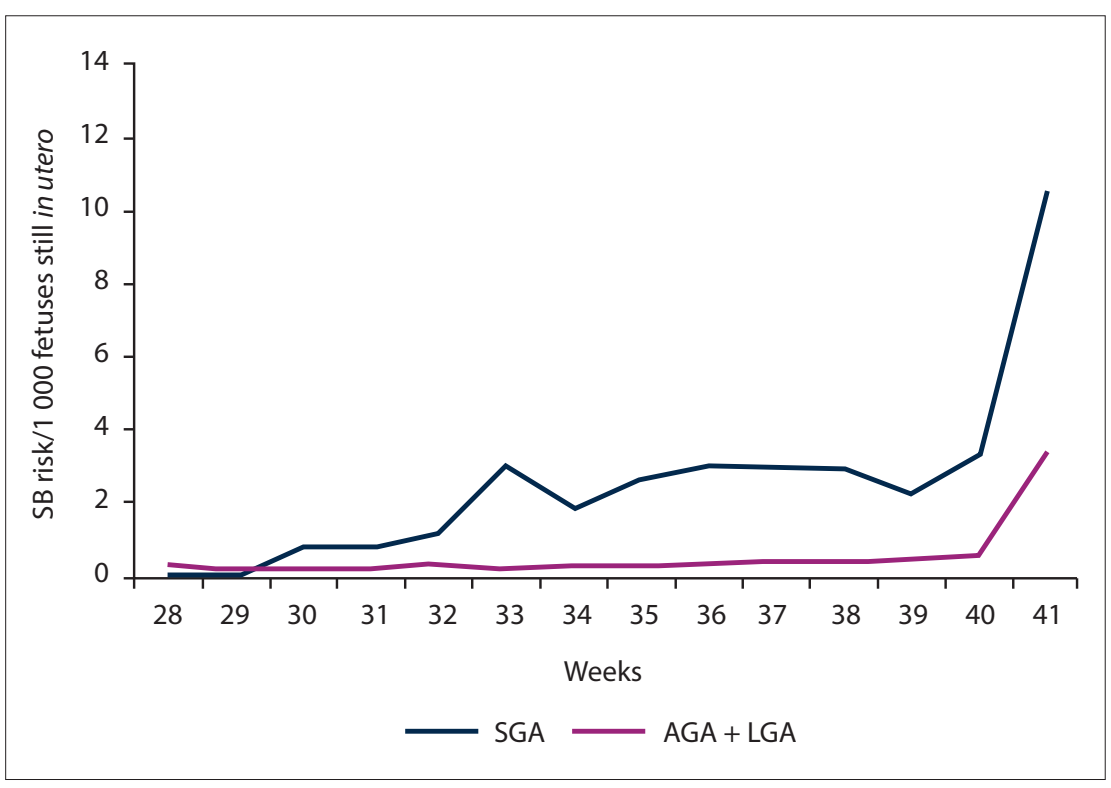

Fig. 4. SB risk across pregnancy in the Western Cape, South Africa, October 2013 - August 2015, using the US cohort distribution of live births (SGA v. non-SGA). ${ }^{[3]}$ (SB = stillbirth; SGA = small for gestational age; $A G A=$ appropriate for gestational age; $L G A=$ large for gestational age.)

pregnancies and SGA infants falling into the 3rd - 10th centiles. ${ }^{[3]}$ Both SGA and AGA pregnancies had an increase in stillbirth risk at 41 weeks. Our findings are consistent with this pattern, where SGA pregnancies had a greater risk than AGA pregnancies, with a rapid increase at 41 weeks. Previous studies have not differentiated between AGA and LGA pregnancies. ${ }^{[3,23,24]}$

Previous work has shown that stillbirth risk is increased during periods without fortnightly ANC visits during this time. This finding highlights the challenges experienced in detecting and managing SGA fetuses, despite women attending ANC. In highincome countries, it is estimated that up to $76 \%$ of SGA cases can be detected during the antenatal period; ${ }^{[25]}$ however, other studies have reported on difficulties of detecting IUGR. ${ }^{[12]}$ Limited resources in LMICs mean that palpation and fundal height are commonly used methods to predict growth restriction, despite little evidence to support their effectiveness. ${ }^{[10]}$ The detection of fetuses at risk of stillbirth is further complicated by the fact that $\sim 50 \%$ occur in a seemingly healthy mother, with no increase in the proportion of unhealthy mothers in SGA pregnancies. ${ }^{[9]}$ Early antenatal detection of SGA babies is important, as most deaths occur in the late preterm or term period when the chance of survival for liveborn infants in well-resourced units is high. ${ }^{[26]}$

A commonly used detection method for SGA in high-resource settings is Doppler velocimetry, which measures umbilical blood flow in high-risk women. ${ }^{[27]}$ This method can increase the ability to detect fetuses with pathological growth restriction and reduce perinatal mortality in high-risk women ${ }^{[28]}$ Doppler velocimetry conducted at earlier gestations has poor predictive value in detecting late-onset placental insufficiency. ${ }^{[29]}$ In SA, significant challenges persist with Doppler measurement, as a large number of perinatal deaths occur in healthy mothers who are clinically considered low risk $^{[13]}$ and are therefore not referred for Doppler screening. A simpler alternative to umbilical artery Doppler is the continuouswave Doppler analyser using a personal computer (Umbiflow), which may be more suitable in the SA context. ${ }^{[30,31]}$ The Umbiflow is able to detect fetuses at risk of stillbirth based on abnormal umbilical arterial blood flow. SA studies have demonstrated that it can be used at primary healthcare level by nurses and midwives. ${ }^{[30,31]}$ Although a recent meta-analysis in high-income countries found that Doppler or Umbiflow use in low-risk pregnancies was not effective in reducing perinatal mortality, ${ }^{[32]}$ there may be a potential benefit of Umbiflow use in lowrisk populations in LMICs to refer women to higher-level care. ${ }^{[33]}$

Another interesting finding in the present study was the increased stillbirth risk seen in LGA pregnancies between 36 and 40 weeks. Few studies have investigated the stillbirth risk across pregnancy for LGA pregnancies, with most research focusing on SGA fetuses. Studies to date have found that LGA infants 
Table 3. Sensitivity analysis comparing stillbirth risk across pregnancy using Mamelodi subdistrict, South Africa, to calculate the denominator compared with US data

\begin{tabular}{|c|c|c|c|c|}
\hline Gestation (weeks) & Mamelodi cohort, RR ( $95 \%$ CI) & $p$-value & US cohort, ${ }^{[3]}$ RR (95\% CI) & $p$-value \\
\hline 28 & $0.2(0.0-2.9)$ & 0.221 & $0.2(0.0-2.8)$ & 0.218 \\
\hline 29 & $0.2(0.0-3.4)$ & 0.267 & $0.2(0.0-3.3)$ & 0.263 \\
\hline 30 & $2.9(1.4-6.1)$ & 0.005 & $2.8(1.4-6.0)$ & 0.006 \\
\hline 31 & $3.3(1.6-7.1)$ & 0.002 & $3.3(1.5-7.0)$ & 0.002 \\
\hline 32 & $3.9(2.1-7.5)$ & $<0.001$ & $3.9(2.0-7.4)$ & $<0.001$ \\
\hline 33 & $13.3(7.9-22.5)$ & $<0.001$ & $13.1(7.7-22.1)$ & $<0.001$ \\
\hline 34 & $6.7(3.8-11.8)$ & $<0.001$ & $6.6(3.7-11.6)$ & $<0.001$ \\
\hline 35 & $9.1(5.4-15.2)$ & $<0.001$ & $8.9(5.3-14.9)$ & $<0.001$ \\
\hline 36 & $8.4(5.2-13.6)$ & $<0.001$ & $8.2(5.1-13.3)$ & $<0.001$ \\
\hline 37 & $6.3(4.0-10.1)$ & $<0.001$ & $6.2(3.9-9.9)$ & $<0.001$ \\
\hline 38 & $6.2(3.8-10.0)$ & $<0.001$ & $6.0(3.7-9.7)$ & $<0.001$ \\
\hline 39 & $4.3(2.4-7.7)$ & $<0.001$ & $4.2(2.3-7.5)$ & $<0.001$ \\
\hline 40 & $5.2(3.0-9.0)$ & $<0.001$ & $5.1(3.0-8.8)$ & $<0.001$ \\
\hline 41 & $3.2(0.9-11.2)$ & 0.0756 & $2.6(0.7-9.2)$ & 0.146 \\
\hline
\end{tabular}

$(>4500 \mathrm{~g})$ are at higher risk of stillbirth than infants weighing $2500-4500 \mathrm{~g}$, but the risk across gestation has not been examined. In $\mathrm{SA}$, the proportion of stillbirths for AGA and LGA babies was spread evenly across gestation, with one-third in each of the $28-32$ weeks, 33 - 37 weeks and 38 - 42 weeks gestational age categories. ${ }^{[9]}$ The increase in LGA stillbirth risk between 36 and 40 weeks may be explained by undiagnosed gestational diabetes, which would present as late stillbirths in LGA pregnancies. Healthy LGA babies would experience more obstructed labour from 40 weeks, resulting in increased mortality risk.

\section{Study strengths and limitations}

The strengths of this audit are that it used whole-population data from a real-life setting and that it allowed for the comparison of SGA/AGA/LGA pregnancies, for which data are scarce in lowresource settings. One limitation to the study is that owing to the association between growth restriction and preterm birth, we expect a greater proportion of preterm births to be SGA because of this association alone. This association introduces issues when using the FAR approach, as a greater proportion of births will be SGA at earlier gestations. We sought to overcome this problem by using the denominator of SGA intrauterine pregnancies, but this is not without its own limitations. The data were cross-sectional, with only one data point for each stillbirth case, which introduces temporal challenges. First, growth restriction is defined by a longitudinal pattern of faltering growth that could not be taken into account in the current analysis. Second, many late stillbirths may not have been SGA before the fetus began to experience growth restriction.

As with all research that explores gestational age-specific associations between exposures and perinatal outcome, collider bias may be present. ${ }^{[34]}$ There is debate in the literature surrounding the desire to obtain gestational age-specific associations and awareness that conditioning on such variables can give rise to bias. ${ }^{[34-36]}$ In the current analysis, associations at lower gestations may appear weaker than at later gestational ages owing to collider bias. The results must therefore be interpreted with caution and in light of the other available evidence.

\section{Conclusions}

The results from this study indicate that stillbirth risk was increased for SGA pregnancies from 33 weeks' gestation compared with AGA pregnancies, despite women having fortnightly ANC appointments during this time. This finding suggests that there is an issue with the detection of fetuses at risk of stillbirth rather than an issue of access to ANC itself. The further investigation of possible methods to detect fetuses at risk of stillbirth in low-risk women suitable for the lowresource setting is warranted and necessary.

Declaration. This publication formed part of TL's PhD degree.

Acknowledgements. None.

Author contributions. TL conceptualised the study, analysed data, drafted the manuscript and edited the manuscript; RCP revised/edited the manuscript; LN assisted with data analysis and interpretation of results; SG revised/edited the manuscript; DP supervised data analysis and revised/edited the manuscript.

Funding. This project was supported by a University of Western Australia Research Collaboration Award. The University of Western Australia, Research Collaboration Award funded travel for this collaboration. The South African Medical Research Council funds the Perinatal Problem Identification Program. TL is funded by an Australian Postgraduate Award.

Conflicts of interest. None.

1. Katz J, Lee AC, Kozuki N, et al. Mortality risk in preterm and small-for-gestational-age infants in lowincome and middle-income countries: A pooled country analysis. Lancet 2013;382(9890):417-425. https://doi.org/10.1016/S0140-6736(13)60993-9

2. Gardosi J, Mul T, Mongelli M, Fagan D. Analysis of birthweight and gestational age in antepartum stillbirths. Br J Obstet Gynaecol 1998;105(5):524-530. https://doi.org/10.1111/j.1471-0528.1998. tb10153.x

3. Pilliod RA, Cheng YW, Snowden JM, Doss AE, Caughey AB. The risk of intrauterine fetal death in the small-for-gestational-age fetus. Am J Obstet Gynecol 2012;207(4):318.e1-318.e6. https://doi. org/10.1016/j.ajog.2012.06.039

4. Lindqvist PG, Molin J. Does antenatal identification of small-for-gestational age fetuses significantly improve their outcome? Ultrasound Obstet Gynecol 2005;25(3):258-264. https://doi.org/10.1002/ uog.1806

5. Bernstein IM, Horbar JD, Badger GJ, Ohlsson A, Golan L, Network VO. Morbidity and mortality among very-low-birth-weight neonates with intrauterine growth restriction. Am J Obstet Gynecol among very-low-birth-weight neonates with intrauterine growth restic

6. Redline RW, Wilson-Costello D, Hack M. Placental and other perinatal risk factors for chronic lung disease in very low birth weight infants. Pediatr Res 2002;52(5):713-719. http://doi. org/10.1203/00006450-200211000-00017

. De Courcy-Wheeler RHB, Wolfe CDA, Warburton F, Goodman J, Reynolds F, Gamsu H. The association between small size for gestational age and perinatal and neonatal death in a UK Regional Health Authority. Paediatr Perinat Epidemiol 1995;9(4):431-440. https://doi.org/10.1111/j.1365-3016.1995. tb00166.x

8. Williams RL, Creasy RK, Cunningham GC, Hawes WE, Norris FD, Tashiro M. Fetal growth and perinatal viability in California. Obstet Gynecol 1982;59(5):624-632.

9. Lavin T, Preen DB, Pattinson R. Timing and cause of perinatal mortality for small-for-gestationalage babies in South Africa: Critical periods and challenges with detection. Matern Health Neonatol Perinatol 2016;2:11. https://doi.org/10.1186/s40748-016-0039-4 
10. Peter JR, Ho JJ, Valliapan J, Sivasangari S. Symphysial fundal height (SFH) measurement in pregnancy for detecting abnormal fetal growth. Cochrane Database Syst Rev 2015, Issue 9. Art. No.: CD008136. for detecting abnormal fetal growth. Cochrane Dat
https://doi.org/10.1002/14651858.CD008136.pub3

11. McKenna D, Tharmaratnam S, Mahsud S, Bailie C, Harper A, Dornan J. A randomized trial using McKenna D, Tharmaratnam S, Mahsud S, Bailie C, Harper A, Dornan J. A randomized trial using
ultrasound to identify the high-risk fetus in a low-risk population. Obstet Gynecol 2003;101(4):626632. https://doi.org/10.1016/S0029-7844(02)03122-8

12. Monier I, Blondel B, Ego A, Kaminski M, Goffinet F, Zeitlin J. Does the presence of risk factors for fetal growth restriction increase the probability of antenatal detection? A French national study. Paediatr Perinat Epidemiol 2016;30(1):46-55. https://doi.org/10.1111/ppe.1225

13. Allanson ER, Muller M, Pattinson RC. Causes of perinatal mortality and associated maternal complications in a South African province: Challenges in predicting poor outcomes. BMC Pregnancy Childbirth 2015;15:37. https://doi.org/10.1186/s12884-015-0472-9

14. Vogel JP, Habib NA, Souza JP, et al. Antenatal care packages with reduced visits and perinatal mortality: A secondary analysis of the WHO Antenatal Care Trial. Reprod Health 2013;10:20. https://doi. org/10.1186/1742-4755-10-20

15. Dowswell T, Carroli G, Duley L, et al. Alternative versus standard packages of antenatal care for low-risk pregnancy. Cochrane Database Syst Rev 2015, Issue 7. Art. No.: CD000934. https://doi. org/10.1002/14651858.CD000934.pub3

16. Lavin T, Pattinson RC. Does antenatal care timing influence stillbirth risk in the third trimester? A secondary analysis of perinatal death audit data in South Africa. BJOG 2018;125(2):140-147. https:// doi.org/10.1111/1471-0528.14645

17. Allanson ER, Pattinson RC. Quality-of-care audit and perinatal mortality in South Africa. Bull World Health Organ 2015;93:424-428. https://doi.org/10.2471/BLT.14.144683

18. Theron GB, Thompson ML. A centile chart for birth weight for an urban population of the Western Cape. S Afr Med J 1995;85(12):1289-1292.

19. Theron GB, Geerts L, Thompson ML, Theron AM. A centile chart for fetal weight for gestational ages 24 - 27 weeks. S Afr Med J 2008;98(4):270-271.

20. Platt RW, Joseph KS, Ananth CV, Grondines J, Abrahamowicz M, Kramer MS. A proportional hazards model with time-dependent covariates and time-varying effects for analysis of fetal and infant death. Am J Epidemiol 2004;160(3):199-206. https://doi.org/10.1093/aje/kwh201

21. Yudkin PL, Wood L, Redman CWG. Risk of unexplained stillbirth at different gestational ages. Lancet 1987;1(8543):1192-1194. https://doi.org/10.1016/s0140-6736(87)92154-4

22. Basso O. Implications of using a fetuses-at-risk approach when fetuses are not at risk. Paediatr Perinat Epidemiol 2016;30(1):3-10. https://doi.org/10.1111/ppe.12254

23. Trudell AS, Cahill AG, Tuuli MG, Macones GA, Odibo AO. Risk of stillbirth after 37 weeks in pregnancies complicated by small-for-gestational-age fetuses. Am J Obstet Gynecol 2013:208(5):376. e1-376.e7. https://doi.org/10.1016/.j.ajog.2013.02.030

24. Rosenstein MG, Snowden JM, Cheng YW, Caughey AB. The mortality risk of expectant management compared with delivery stratified by gestational age and race and ethnicity. Am J Obstet Gynecol 2014;211(6):660.e1-660.e8. https://doi.org/10.1016/.ajog.2014.06.008
25. Pearce JM, Campbell S. A comparison of symphysis-fundal height and ultrasound as screening tests for light-for-gestational age infants. Br J Obstet Gynaecol 1987;94(2):100-104. https://doi org/10.1111/j.1471-0528.1987.tb02333.x

26. Velaphi SC, Mokhachane M, Mphahlele RM, Beckh-Arnold E, Kuwanda ML, Cooper PA. Survival of very-low-birth-weight infants according to birth weight and gestational age in a public hospital. $\mathrm{S}$ Af Med J 2005;95(7):504-509.

27. Reuwer PJ, Bruinse HW, Stoutenbeek P, Haspels AA. Doppler assessment of the fetoplacenta circulation in normal and growth-retarded fetuses. Eur J Obstet Gynecol Reprod Biol 1984;18(4):199205. https://doi.org/10.1016/0028-2243(84)90117-5

28. Alfirevic Z, Stampalija T, Dowswell T. Fetal and umbilical Doppler ultrasound in high-risk pregnancies. Cochrane Database Syst Rev 2017. Issue 6. Art. No. CD007529. https://doi.org/10.1002/14651858. CD007529.pub4

29. Figueras F, Caradeux J, Crispi F, Eixarch E, Peguero A, Gratacos E. Diagnosis and surveillance of late-onset fetal growth restriction. Am J Obstet Gynecol 2018;218(2):S790-S802.e1. https://doi org/10.1016/j.ajog.2017.12.003

30. Hugo EJC, Odendaal HJ, Grove D. Evaluation of the use of umbilical artery Doppler flow studies an outcome of pregnancies at a secondary hospital. J Matern Fetal Neonatal Med 2007;20(3):233-239. https://doi.org/10.1080/14767050601134926

31. Theron GB, Theron AM, Odendaal HJ, Bunn AE. Comparison between a newly developed PC-based Doppler umbilical artery waveform analyser and a commercial unit. S Afr Med J 2005;95(1):62-64.

32. Alfirevic Z, Stampalija T, Medley N. Fetal and umbilical Doppler ultrasound in normal pregnancy. Cochrane Database Syst Rev 2015, Issue 4. Art. No: CD001450. https://doi.org/10.1002/14651858. CD001450.pub4

33. Mufenda J, Gebhardt S, van Rooven R, Theron G. Introducing a mobile-connected umbilical Doppler device (UmbiFlow) into a primary care maternity setting: Does this reduce unnecessary referrals to specialised care? Results of a pilot study in Kraaifontein, South Africa. PLoS One 2015;10(11):e0142743 https://doi.org/10.1371/journal.pone.0142743

34. VanderWeele TJ, Mumford SL, Schisterman EF. Conditioning on intermediates in perinata epidemiology. Epidemiology 2012;23(1):1-9. https://doi.org/10.1097/EDE.0b013e31823aca5d

35. Wilcox AJ, Weinberg CR, Basso O. On the pitfalls of adjusting for gestational age at birth. Am Epidemiol 2011;174(9):1062-1068. https://doi.org/10.1093/aje/kwr230

36. Whitcomb BW, Schisterman EF, Perkins NJ, Platt RW. Quantification of collider-stratification bias and the birthweight paradox. Paediatr Perinat Epidemiol 2009;23(5):394-402. https://doi.org/10.1111/ j.1365-3016.2009.01053.x

Accepted 14 June 2019 